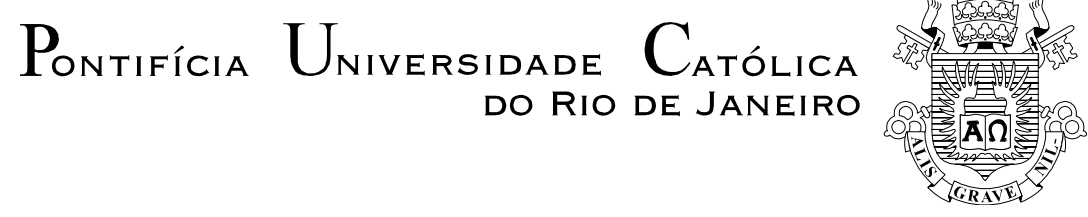

Sandoval José Rodrigues Júnior

\author{
Otimização de Pilares \\ de Edifícios Altos de Concreto Armado
}

Tese de Doutorado

Tese apresentada como requisito parcial para obtenção do título de Doutor pelo Programa de PósGraduação em Engenharia Civil da PUC-Rio.

Orientadores: Prof. Giuseppe Barbosa Guimarães

Prof. Luiz Eloy Vaz

Rio de Janeiro, setembro de 2005 


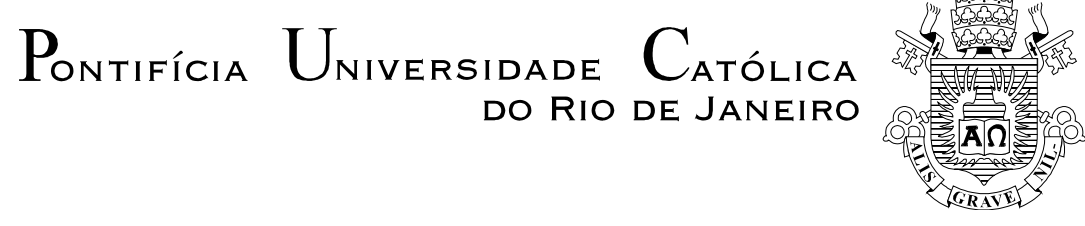

Sandoval José Rodrigues Júnior

\title{
Otimização de Pilares de Edifícios Altos de Concreto Armado
}

Tese apresentada como requisito parcial para obtenção do título de Doutor pelo Programa de Pós-Graduação em Engenharia Civil da PUC-Rio. Aprovada pela Comissão Examinadora abaixo assinada.

\author{
Prof. Giuseppe Barbosa Guimarães \\ Orientador \\ Departamento de Engenharia Civil - PUC-Rio
}

Prof. Luiz Eloy Vaz

Co-orientador

UFRJ

Prof. Raul Rosas e Silva

Departamento de Engenharia Civil - PUC-Rio

Profa. Marta de Souza Lima Velasco

Departamento de Engenharia Civil - PUC-Rio

Profa. Cláudia Ribeiro Eboli

UFRJ

Prof. Ibrahim Abd Ei Malik Shehata

UFRJ

Prof. José Eugênio Leal

Coordenador Setorial do Centro Técnico Científico - PUC-Rio

Rio de Janeiro, 16 de setembro de 2005 
Todos os direitos reservados. É proibida a reprodução total ou parcial do trabalho sem autorização da universidade, do autor e do orientador.

\section{Sandoval José Rodrigues Júnior}

Graduou-se em Engenharia Civil na UFPA/Belém (Universidade Federal do Pará) em 1988. Concluiu o mestrado na PUC-Rio em 1996. Interesses acadêmicos em áreas de pesquisa que envolvam concreto armado, otimização de estruturas, programação matemática. Atualmente é professor do Departamento de Engenharia Civil da UFPA.

Ficha Catalográfica

Rodrigues Júnior, Sandoval José

Otimização de Pilares de Edifícios Altos de Concreto Armado / Sandoval José Rodrigues Júnior; orientador: Giuseppe Barbosa Guimarães; co-orientador: Luiz Eloy Vaz. - Rio de Janeiro : PUC, Departamento de Engenharia Civil, 2005.

v., 154 f.: il. ; $29,7 \mathrm{~cm}$

1. Tese (Doutorado) - Pontifícia Universidade Católica do Rio de Janeiro, Departamento de Engenharia Civil.

Incluí referências bibliográficas.

1. Engenharia Civil - Teses. 2. Otimização. 3. Análise de sensibilidade. 4. Programação matemática. 5. Projeto ótimo. 6. Pórtico espacial. 7. Concreto armado. 8. Edifícios altos. I. Guimarães, G. B. (Giuseppe B.). II. Vaz, L. E. (Luiz Eloy). III. Pontifícia Universidade Católica do Rio de Janeiro. IV. Título 


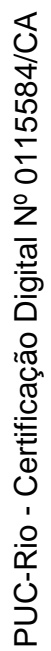

Dedicado aos meus pais, Sandoval e Lucyléa Rodrigues 


\section{Agradecimentos}

Aos meus orientadores Giuseppe Barbosa Guimarães e Luiz Eloy Vaz pela orientação e amizade.

Aos professores do Departamento de Engenharia Civil da PUC-Rio, em especial ao professor João Luis Pascal Roehl, por todos os conhecimentos transmitidos durante a pós-graduação.

À minha esposa Lucilene, e minhas filhas Caroline e Bruna, por todo o amor, carinho, e companheirismo que muito contribuíram para a realização deste trabalho.

A toda minha família, em especial aos meus pais e meus irmãos Sandra, Jefferson e Daniele.

Aos grandes amigos Anderson e Müller, pelas excelentes discussões ao longo deste trabalho.

A todos os amigos da PUC, em especial Galvão, Ivy, Joabson, Antonio Sérgio, Antônio Miranda, Chan, Walter, Maurício, Pasquetti, Andréia e Denyse.

Aos funcionários da PUC-Rio, em particular a Ana Roxo, Lenilson, Cristiano, Euclides, Haroldo, José Nilson e Evandro.

Aos professores do Departamento de Engenharia Civil da UFPA, em especial ao grande amigo José Raimundo Serra Pacha, pelas discussões e sugestões ao presente trabalho.

À CAPES, à UFPA e à PUC-Rio, pelos auxílios concedidos. 


\section{Resumo}

Rodrigues Júnior, Sandoval José; Guimarães, Giuseppe Barbosa; Vaz, Luiz Eloy. Otimização de Pilares de Edifícios Altos de Concreto Armado. Rio de Janeiro, 2005. 154p. Tese de Doutorado - Departamento de Engenharia Civil, Pontifícia Universidade Católica do Rio de Janeiro.

O presente trabalho propõe uma formulação para o projeto ótimo de pilares de edifícios altos de concreto armado. São variáveis de projeto as dimensões da seção transversal e a armadura longitudinal dos pilares e a resistência característica do concreto. A fim de reduzir o tamanho desta classe de problema, a estratégia de solução em multinível é aplicada. O problema é então subdividido em um problema global de otimização conectado a uma série de subproblemas individuais de otimização. No problema global são determinadas as dimensões da seção transversal de todos os pilares e a resistência característica do concreto, enquanto que nos subproblemas individuais são determinadas apenas as armaduras longitudinais dos pilares. As variáveis dos subsistemas são freqüentemente chamadas de variáveis locais, enquanto que as variáveis do sistema global, responsáveis pela interação entre os grupos de variáveis, são denominadas variáveis globais ou de acoplamento. A função objetivo do problema de otimização é o custo total das colunas do edifício. Os edifícios são modelados como pórticos espaciais e a não-linearidade geométrica é considerado na análise estrutural. A hipótese de diafragma rígido é adotada para o comportamento das lajes e a rigidez à torção é desprezada em todas as barras do pórtico. Cargas permanentes, acidentais e devidas ao vento são aplicadas ao modelo. Restrições relativas aos estados limite último e de utilização, bem como restrições relativas aos limites máximos e mínimos atribuídos a cada variável, são impostas ao problema de otimização. Adicionalmente, impõem-se restrição sobre o parâmetro de instabilidade, caso este parâmetro seja empregado na determinação dos esforços globais de 2a ordem. Os estados limite são definidos de acordo com o código brasileiro para projeto de estruturas de concreto NBR-6118 (2003). O problema de otimização proposto é resolvido empregando-se técnicas de programação matemática. 


\section{Palavras-chave}

Otimização; análise de sensibilidade; programação matemática; projeto ótimo; pórtico espacial; concreto armado; edifícios altos. 


\section{Abstract}

Rodrigues Júnior, Sandoval José; Guimarães, Giuseppe Barbosa; Vaz, Luiz Eloy (advisors). Optimization of Columns of Reinforced Concrete Tall Buildings. Rio de Janeiro, 2005. 154p. D.Sc. Thesis - Departamento de Engenharia Civil, Pontifícia Universidade Católica do Rio de Janeiro.

This work proposes a formulation for the optimum design of columns of reinforced concrete tall buildings. The design variables are the dimensions of the cross-sections and the amount of longitudinal reinforcement in the columns and the concrete characteristic resistance. Aiming at reducing the size of this class of problem, the multilevel optimization technique is applied so that the problem is subdivided into a global problem connected with a series of dependent subproblems. In this kind of problem, the design variables are subdivided into two groups, namely, the group of the so called global variables, represented here by the cross-section dimensions of the columns and by the concrete characteristic resistance, and the group of local variables, represented here by the areas of the longitudinal steel reinforcement in the cross-sections of the columns. Consequently, a global optimization problem is defined for the global variables, at the global level, and local independent optimization sub-problems are defined for the local design variables, at the local levels, following each iteration at the global level. The objective function of the optimization problem is the total cost of the columns of the building. The buildings are modeled as three dimensional frames and geometric nonlinear behavior is considered in the structural analysis. The assumption of rigid diaphragm is adopted for the structural behavior of the slabs and the torsional stiffness of elements is neglected. Dead, live and wind loads are applied to the model. Constraints related to ultimate and serviceability limit states, as well as lateral constraints, are imposed to the design variables. The limit states are defined according to the Brazilian Code for reinforced concrete structures NBR-6118 (2003). The solution of the optimization problem is obtained using Mathematical Programming techniques. 


\section{Keywords}

Optimization; sensibility analysis; mathematical programming; optimal design; three-dimensional frame; reinforced concrete; tall buildings. 


\section{SUMÁRIO}

$\begin{array}{ll}\text { 1. Introdução } & 16\end{array}$

1.1. Considerações Gerais 16

1.2. Revisão Bibliográfica 18

1.3. Objetivos e Justificativas 26

1.4. Escopo do Trabalho 29

2. Contraventamento de Edifícios de Concreto Armado 31

2.1. Parâmetros de Instabilidade 33

2.1.1. Parâmetros de Instabilidade $\alpha \quad 34$

2.1.2. Parâmetros de Instabilidade $\gamma_{Z} \quad 35$

2.2. Rigidez Limite do Sistema de Contraventamento 37

2.3. Projetos: Processos Ótimos e Tradicionais $\quad 37$

3. Modelo e Análise Estrutural 40

3.1. Modelo Estrutural 40

3.1.1. Esforços de Eng. Perfeito e vão Efetivo das Vigas 40

3.2. Análise Estrutural 42

3.2.1. Não-Linearidade Física 42

3.2.2. Não-Linearidade Geométrica 44

3.2.3. Combinações e Fatores de Ponderação das Ações 50

3.2.4. Simulação das Lajes como pavimento Rígido 54

$\begin{array}{ll}\text { 3.2.5. Estratégia de Solução } & 57\end{array}$

4. Concreto Armado $\quad 59$

4.1. Dimensionamento à Flexão Composta Oblíqua 59

4.1.1. Características mecânicas dos materiais 59

4.1.2. Esforços Atuantes 61

4.1.3. Parâmetros de Descrição da Deformada da Seção 61

4.1.4. Esforços Resistentes de uma Seção 64

4.1.5. Dimensionamento de uma Seção 66

4.2. Dimensionamento de Pilares segundo a NBR 6118:2003 67

4.2.1. Classificação quanto à Esbeltez 68 
4.2.3. Momento Mínimo 70

4.2.4. Imperfeições Geométricas Locais 70

$\begin{array}{ll}\text { 4.2.5. Efeitos Locais de Segunda Ordem } & 71\end{array}$

4.2.6. Distribuição da Armadura Longitudinal As na seção 75

4.2.7. Comprimento de Ancoragem da Armadura Longitudinal 76

4.2.8. Armadura Transversal $\quad 77$

5. Programação Matemática - Princípios Básicos 78

5.1. Considerações Gerais 78

5.2. Condições de Ótimo $\quad 79$

5.3. Forma Geral dos Algoritmos de Otimização 80

5.4. Método de Newton para Problemas de otimização s/ Restrição 81

5.5. Busca Linear 82

5.6. Programação Quadrática 83

5.7. Algoritmo de Han-Powell - Programação Quadrática Seqüencial 84

5.7.1.Etapas do Algoritmo Não-Linear Han-Powell (PQS) 86

5.8. Método dos Pontos Interiores 88

5.8.1.Etapas do Algoritmo de Pontos Interiores 90

6. Otimização de Dimensões 93

6.1. Considerações Gerais 93

6.2. Otimização em Multinível 93

6.3. Metodologia de Otimização 96

6.4. Formulação do Problema de otimização 100

6.4.1. Função Objetivo 101

6.4.2. Restrições Relativas à Resistência 101

6.4.3. Limites das Armaduras Longitudinais 102

6.4.4. Estado Limite de Deformação Excessiva da Estrutura 102

6.4.5. Restrições sobre o parâmetro de Instabilidade Global $\gamma_{z} \quad 103$

$\begin{array}{ll}\text { 6.4.6. Restrições Laterais } & 103\end{array}$

6.4.7. Restrições Laterais Relativas 104

$\begin{array}{ll}\text { 6.4.8. Fatores de Escala } & 104\end{array}$

$\begin{array}{ll}\text { 7. Análise de Sensibilidade } & 107\end{array}$

$\begin{array}{ll}\text { 7.1. Considerações Gerais } & 107\end{array}$ 
7.2. Sensibilidade dos Deslocamentos

7.3. Sensibilidade das Forças Externas $\{P(x)\} \quad 110$

$\begin{array}{ll}\text { 7.3.1. Parcelas devidas ao Peso Próprio } & 110\end{array}$

7.3.2. Parcelas devidas à consideração do vão Efetivo 110

$\begin{array}{ll}\text { 7.3.3. Parcelas devidas ao Parâmetro } \gamma_{z} & 111\end{array}$

7.4. Sensibilidade dos Esforços Internos Solicitantes 112

$\begin{array}{ll}\text { 7.5. Sensibilidade da Armadura } & 114\end{array}$

7.5.1. Sensibilidade da Armadura Longitudinal 114

7.5.2. Sensibilidade da Armadura Transversal 115

7.6. Sensibilidade dos Esforços Solicitantes de Projeto 116

7.6.1. Seção Crítica nos Extremos do Pilares 116

7.6.2. Seção Crítica no Centro dos Pilares 116

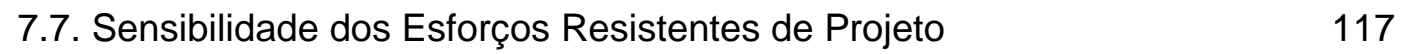

$\begin{array}{ll}\text { 8. Implementações Computacionais e Exemplos } & 119\end{array}$

$\begin{array}{ll}\text { 8.1. Implementações Computacionais } & 119\end{array}$

$\begin{array}{ll}\text { 8.2. Exemplos } & 121\end{array}$

8.2.1. Materiais: Propriedades Mecânicas e Custos 121

8.2.2. Exemplo I 122

$\begin{array}{ll}\text { 8.2.3. Exemplo II } & 135\end{array}$

9. Conclusões e Sugestões 148

$\begin{array}{ll}\text { 9.1. Conclusões } & 148\end{array}$

$\begin{array}{ll}\text { 9.2. Sugestões } & 149\end{array}$

$\begin{array}{ll}\text { 10. Referências Bibliográficas } & 150\end{array}$

ANEXO A - Fatores de Ponderação e Combinação 154 


\section{Lista de figuras}

Figura 2.1 - Sistema associado de pórticos e paredes estruturais

Figura 3.1 - Vão efetivo das vigas.

Figura 3.2 - Modelo para determinação dos esforços de engastamento

perfeito.

Figura 3.3 - Eixos de referência e graus de liberdade locais.

Figura 3.4 - Imperfeições geométricas globais.

Figura 3.5 - Deslocamentos nodais e distância entre os nós $i$ e j.

Figura 4.1 - Diagrama tensão-deformação idealizado (NBR 6118 (2003))

Figura 4.2 - Diagrama tensão-deformação para aços de armadura passiva (NBR 6118 (2003)).

Figura 4.3 - Esforços atuantes de cálculo.

Figura 4.4 - Inclinação da linha neutra.

Figura 4.5 - Domínios de estado limite último de uma seção transversal (NBR 6118 (2203)).

Figura 4.6 - Esquematização da deformada da seção.

Figura 4.7 - Definição das regiões 0 , I e II da seção.

Figura 4.8 - Lande de pilar - Imperfeições geométricas locais.

Figura 4.9 - Posicionamento de $A s$ e $A s_{w}$ na seção.

Figura 6.1 - Estruturas de decomposição em bloco diagonal e angular.(95)

Figura 6.2 - Esquema para projeto ótimo de estruturas de concreto armado em multinível.

Figura 6.3 - Tipos de seções transversais admitidas para os pilares .. (100)

Figura 8.1 - Planta de fôrma estrutural - Exemplo I:

edifício de 24 pavimentos tipo

Figura 8.2 - Variação do custo dos pilares com o $f_{c k}$

Figura 8.3 - Variação do custo dos pilares com o $f_{c k}$

Figura 8.4 - Planta de fôrma estrutural - Exemplo I: proposta (I)

Figura 8.5 - Planta de fôrma estrutural - Exemplo I: proposta (II). 
Figura 8.6 - Redução relativa do custo dos pilares com respeito a redução de seção.

Figura 8.7 - Redução relativa de material dos pilares com respeito a redução de seção.

Figura 8.8 - Variação relativa de material para o modelo $R(0)$ considerando possíveis variações no custo do concreto.

Figura 8.9 - Planta de fôrma estrutural - Exemplo II:

edifício de 24 pavimentos tipo

Figura 8.10 - Redução relativa do custo dos pilares com respeito a redução de seção.

Figura 8.11 - Redução relativa de material dos pilares com respeito a redução de seção.

Figura 8.12 - Redução relativa do custo dos pilares com respeito a redução de seção: comparação entre os métodos SQP e IP.

Figura 8.13 - Verificação da capacidade resistente do pilar P1, lance1 (opção 1)

Figura 8.14 - Verificação da capacidade resistente do pilar P1, lance1 (opção 2).

Figura 8.15 - Verificação da capacidade resistente do pilar P3, lance1 (opção 1).

Figura 8.16 - Verificação da capacidade resistente do pilar P3, lance1 (opção 2). 


\section{Lista de tabelas}

Tabela 4.1 - Correspondência entre os domínios dos E.L.U.

(NBR 6118 (2003)) e os valores do parâmetro D e das funções $\varepsilon_{S}$ e $\varepsilon_{i}(63)$

Tabela 5.1 - Divisão dos problemas de Programação Matemática

Tabela 8.1 - Preços de concreto massa $\left(R \$ / \mathrm{m}^{3}\right)$.

Tabela 8.2 - Variação do parâmetro de instabilidade $\gamma_{z}$ com o $f_{c k} \ldots$ (125)

Tabela 8.3 - Resultados ótimos $(\mathrm{cm})$ : Classe C35

Tabela 8.4 - Variação do parâmetro $\gamma_{Z}$ com respeito a redução de seção.

Tabela 8.5 - Resultados ótimos para o modelo R(0)

Tabela 8.6 - Resultados ótimos para o modelo $R(1)$

Tabela 8.7 - Resultados ótimos para o modelo R(2)

Tabela 8.8 - Resultados ótimos para o modelo R(3)

Tabela 8.9 - Deslocamentos máximos relativos no topo do prédio

Tabela 8.10 - Variação da taxa de armação com respeito a redução de seção (\%)

Tabela 8.11 - Resultados ótimos para o modelo $\mathrm{R}(0)$ considerando possíveis variações no custo do concreto.

Tabela 8.12 - Variação do parâmetro $\gamma_{z}$ com respeito à redução de seção

Tabela 8.13 - Deslocamentos máximos no topo do prédio

Tabela 8.14 - Resultados ótimos para o modelo $R(0)$

Tabela 8.15 - Resultados ótimos para o modelo $R(1)$

Tabela 8.16 - Resultados ótimos para o modelo R(2)

Tabela 8.17 - Resultados ótimos para o modelo R(3)

Tabela 8.18 - Variação da taxa de armação com a redução de seção (141)

Tabela 8.19 - Tempos de processamento - SQP x IP.

Tabela A.1 - Coeficiente $\gamma_{f}=\gamma_{f 1} \cdot \gamma_{f 3}$ (NBR 6118 (2003))

Tabela A.2 - Valores do coeficiente $\gamma_{f 2}$ (NBR 6118 (2003)) 